\title{
Exploring Current Challenges for Models of Species Distribution and Ecological Niches
}

\author{
Paulo De Marco Júnior* \\ Laboratório de Ecologia Teórica e Síntese, Departamento de Ecologia, Instituto de Ciências Biológicas - ICB, \\ Universidade Federal de Goiás - UFG, Goiânia, GO, Brasil
}

Key words: Bioclimatic Envelope Models, Climate Change, Ecological Niche Models, Ensemble Forecasting, Methodological Issues, Species Distribution Modeling.

\section{Species Distributions and their Models}

"Now is the winter of our discontent made glorious summer..."(Richard III, Shakespeare).

There is an increasingly discontent chorus echoing around methodological issues related to ecological niche modeling and projection of species distributions (Jiménez-Valverde \& Lobo 2006; Peterson et al. 2007; Jiménez-Valverde et al. 2008; Lobo et al. 2008). Methods for modeling ecological niches are ubiquitous in recent ecological literature but, at the same time, this literature reveals a plethora of methodological issues to be addressed. This is exactly how good science is done. Now, increased dissemination of good practices, well-posed questions, and carefully designed procedures (both for data gathering and for model building) help putting these excellent tools in harmonic resonance with a set of theoretical arguments, conceptual models, and a critical evaluation of its data statements. This special issue of "Natureza \& Conservação" is framed in this spirit: to put together some of the practitioners, which had presented important criticism to the use of ecological niche models and, then, help develop this field. Of course - and returning to the epigraph of this text - this simply does not make any sense without a glorious summer at the end.

In several moments, for many different research topics in ecological and evolutionary science, it was possible to devise a rapid development of theoretical and analytical tools attached to a confusing and probably not justified increase of definitions, which, in turn, induce to a multiplication of semantic problems. For instance, in the study of species' distributions based on niche properties, we can see terms like "environmental niche models", "ecological niche models", "species distribution models", "envelope models",

\footnotetext{
*Send correspondence to: Laboratório de Ecologia Teórica e Síntese, Departamento de Ecologia, Instituto de Ciências Biológicas - ICB, Universidade Federal de Goiás - UFG, CP 131, CEP 74001-9700, Goiânia, GO, Brasil

E-mail:pdemarco@icb.ufg.br
}

"bioclimate envelope models", to list a few. In worst cases saying "potential distributions" and "realized niche" may favor erroneous concept translocations leading to non-sense terms such as "potential niche" and "realized distribution". On the other hand, some concept distinctions do not represent a semantic problem, but state a point of view that clarifies its use. This clarification is what we can learn from Peterson \& Soberón, the opening paper of this issue, which deals with a close examination of the widely used terms "species distribution models" (SDMs) and "ecological niche models" (ENMs). Distinction between these two terms represents an obvious first step to use these procedures into a well-known theoretical landscape (see also Araújo \& Peterson 2012). These authors are also directly associated to some of the major theoretical advances in the field (e.g. Soberón \& Peterson 2005; Soberón 2007) and reinforce here the importance to have a strong theoretical and conceptual background to build such models.

Hortal et al. make another profound distinction: they distinguish between ahistorical and historical views of this field. There is no hesitation in saying that contemporary/ climatic factors are important to determine species distribution. But neglecting history may imply in putting aside important factors that help understand the big picture. This is obviously a great challenge - to put history into models - but these authors propose a series of practical choices that can fulfill this job.

Even if we can see more clearly what these models mean after Peterson \& Soberón's and Hortal et al.'s distinctions, all practitioners have to choose a procedure among the large number of different methods now available. They came from different parts of our methodological toolbox, representing statistical procedures for curve fitting, neural-networks, optimization, or simple rules as criteria to predict species distribution or describe species niche properties. Rangel \& Loyola help us by providing a "metaphorical" classification 
of modeling methods that help both in understanding their properties and to establish in which situations we should use one or another. This study highlights the difference in maximizing the understanding of factors that affect species' distribution or maximize the predictive power of models, even if this requires multiplying the number of parameters.

Finally, Raes closes the section of Essays \& Perspectives evaluating the extend scale effect on model outcomes. He shows that modeling using artificial boundaries or restricting model building to a portion of total distribution of a species may produce underestimation of species distribution. These results also highlight the importance to gather the most complete set of occurrence points as possible to initiate a modeling approach.

The second group of papers, published as Research Letters, begins exactly with the issue of data gathering and data quality. De Giovanni et al. show the pitfalls of taking online database of species occurrences as true, presenting a protocol to better deal with this problem. It is important to revive the minds of every practitioner that data quality is essential, especially when number of records is low. The use of protocols and filters as described in De Giovanni et al. should be extended to other groups, and I expect that they will be considered an essential part in the process of accepting a contribution for scientific journals in the future.

After this we have four contributions that try to discuss historical problems, using different methods and subjects. Lima-Ribeiro et al. searched from patterns of past distribution of the giant ground sloths. They contribute for the Pleistocene mass extinction debate and favor the interpretation that climate change may have had an important role in the extinction of these sloths. Terribile et al. also searched for patterns in past climates, but go further to evaluate its similarity with future climates, and propose a statistical method for this analysis. Their findings could be extremely important to understand some evolutionary paths, and to predict what does the future holds for our biodiversity. Premoli et al. coupled phylogeographical methods with ecological niche models to discuss the past history of the distribution of two mountain trees. They show that these cold-tolerant species may have a long history in these areas and faced iced periods with minor range shifts. Collevatti et al. also looked into a near past, but also bring us with some innovative statistical phylogeographic tools - coalescence analysis - that also helps understanding current distribution of genetic variability within a species' range. Moreover, these two last papers show how integrative this field can be: molecular genetics, geographic distribution, demography, and phylogeography can contribute greatly to simultaneously understanding of current patterns in species distribution.

Nabout et al. present a different use for these models: to understand the productivity of maize crops in Brazil facing global change. They also try to evaluate an interesting distinction between correlative and mechanistic models.
We must consider that, if maize productivity is related to habitat quality, these models can connect, even indirectly, the position in the niche space and ecological properties beyond simple species distribution. This is an important question for current species distribution model theory and, at the same time, reinforces the possibility of using ENMs in evaluating agricultural and economic questions.

Another field of intense research is the use of these models to investigate the geographic range dynamics of an invasive species (see Jiménez-Valverde et al. 2011). Vital et al. develop a model for discussing how environmental traits of different sub-species of honeybees inside their original distribution in Africa could explain current distribution of "Africanized" honeybees in the New World.

Contemporary rare species are the opposite problem of invasive species modeling. They are rare in the field, rare in collections, rare in museums, but abundant in "red lists". The following two Research Letters discuss how to model rare species, and with an additional handicap: they are endemic to mountain ridges. Kamino et al. study the distribution of the plant Petunia mantiqueirense, as an example to evaluate current methods for modeling rare, endemic species. They show to us the importance of using simple environmental similarity methods to address these problems and, more important, how to couple modeling and re-sample field areas to produce better and well evaluated results. Serra et al. also try to model a restricted species with an even lower number of records, the stingless bee Melipona capixaba. They explore different ways by which a species can have a small distribution, comparing models with and without incorporation of dispersal limitation. As in Kamino et al., they also propose areas that should be more intensely inventoried for these stingless bees and areas that should be prioritized for their conservation.

Another example of rare species are red bellied toads, endemic of "restinga" habitats of southern Brazil and Uruguay. Following the conservation focus of the previous two studies, Bernardo-Silva et al. compared different modeling techniques to produce a consensus prediction of suitable areas for these species. The original habitat of these species is highly threated and choice of appropriate areas to set-aside as conservation units is the last hope for these toads and to a long list of species associated to these systems.

Two studies tested models for more abundant species. Ferraz et al. studied the distribution of the jaguar (Panthera onca) in the Atlantic Forest, including landscape variables that may be understood as surrogates of human footprint. These models may contribute to a faster evaluation of currently altered areas for conservation purposes. In the same way, Weber \& Grelle present results of an analysis that links suitability derived from niche models to relative abundance of a bat species. The promising results of this article support the use of model results as proxy for abundance, something with fine and important conservation implications. 
Two Forum papers and a book review close this special issue. The forum papers are related to ongoing conservation projects that use niche models and may produce results that could be direct incorporated as government policies in Brazil. Tôrres \& Vercillo present the view from the Instituto Chico Mendes (ICMBio) on the use of these models in vulnerability assessments. They also highlight the need for scientists and government analysts to establish a better communication channel and that such models must be presented in such a way that they can be readily used by governmental agencies. From another point of view, Paglia et al. discuss how we need to develop strategies for climate change adaptation presenting an ecosystem adaptation program that could be developed using analysis of climate vulnerability delivered by species distribution models. They present the "IKI" project, an initiative captained by the NGO "Conservation International", which combined a variety of different researchers, with different background, around the same goal of generating knowledge for more efficient adaptation to future (or current) climate changes. Finally, the new book by Peterson et al. (2011), reviewed by Diniz-Filho \& Loyola, may be a fine starting point for a conceptual and methodological unification of the field and thus can be considered an important and obligatory reading for those interested in ENMs and SDMs and their applications in Ecology, Biogeography and Conservation Biology.

\section{A Final Remark}

Modeling species' niche and projecting their distribution is important for ecologists (interested in theoretical questions), conservationists (looking into practical problems), biogeographers (searching for distributional patterns), and especially for scientists that try to put all this together. As our general knowledge increase, we expect that new methodological questions arise. Nevertheless, from many published papers in this issue it is possible to foresee that a more careful treatment of the basic hard data for such enterprises - the record of species distribution - is our most important current action. Some models in this issue focused on rare species and need to be considered as an incentive and a guide to get back to the field and execute a well-designed search for novel occurrences. Models are important, but even more is good data. The important thing here is that you can have one (the model) to improve the other (the data).

Challenges for improving these models are expected to change continuously, or this may become a dead field. New scales, the plea for new climatic variables, better way to determine which spatial resolution is important to each problem, better way to choose procedures and variables when dealing with multi-species problems, and probably one of the most important issues, how to include dispersal limitation into our final results. Some of these problems were discussed here, some of them we expect to return in the near future to the pages of "Natureza \& Conservação", as forthcoming papers. The bottom line is that we expect this issue stimulate many scientist currently working on these models both to show their findings here, and to reflect our main proposal to be a ready and fast-publishing vehicle for the study of biodiversity, based on general tools and focusing on the development of a solid methodological and conceptual background as a basis for conservation actions.

\section{Acknowledgements}

I would like to thank several anonymous reviewers that help to keep this issue in the right track of the schedule. A special thanks to J. A. F. Diniz-Filho, who provided intellectual insights and helped in all phases of the assembly of this special issue. I would also to thank C. Nóbrega, N. M. Tôrres, R. Dobrovolski, F. Villalobos, D. Paiva-Silva, and S. Gouveia, they all accompanied side-by-side the development of these ideas. I also thank to $\mathrm{CNPq}$ for providing continuous research productivity grants to my research.

\section{References}

Araújo MB \& Peterson AT, 2012. Uses and misuses of bioclimatic envelope modelling. Ecology, 93:1527-1539. PMid:22919900. http://dx.doi.org/10.1890/11-1930.1

Jiménez-Valverde A \& Lobo JM, 2006. The ghost of unbalanced species distribution data in geographical model predictions. Diversity and Distributions, 12:521-524. http://dx.doi. org/10.1111/j.1366-9516.2006.00267.x

Jiménez-Valverde A, Lobo JM \& Hortal J, 2008. Not as good as they seem: the importance of concepts in species distribution modelling. Diversity and Distributions, 14:885-890. http:// dx.doi.org/10.1111/j.1472-4642.2008.00496.x

Jiménez-Valverde A et al., 2011. Use of niche models in invasive species risk assessments. Biological Invasions, 13:2785-2797. http://dx.doi.org/10.1007/s10530-011-9963-4

Lobo JM, Jiménez-Valverde A \& Real R, 2008. AUC: a misleading measure of the performance of predictive distribution models. Global Ecology and Biogeography, 17:145-151. http://dx.doi.org/10.1111/j.1466-8238.2007.00358.x

Peterson AT, Papes M \& Eaton M, 2007. Transferability and model evaluation in ecological niche modeling: a comparison of GARP and Maxent. Ecography, 30:550-560. http://dx.doi. org/10.1111/j.0906-7590.2007.05102.x

Peterson AT et al., 2011. Ecological Niches and Geographic Distributions. Princeton: Princeton University Press.

Soberón J, 2007. Grinnellian and Eltonian niches and geographic distributions of species. Ecology Letters, 10:1115-1123. PMid:17850335. http://dx.doi. org/10.1111/j.1461-0248.2007.01107.x

Soberón J \& Peterson AT, 2005. Interpretation of models of fundamental ecological niches and species' distributional areas. Biodiversity Informatics, 2:1-10. 